\title{
Modes interplay controls the second harmonic generation dynamics of plasmonic nanostructures
}

Kiselev, Andrei, Bernasconi, Gabriel, Martin, Olivier J.

Andrei Kiselev, Gabriel D. Bernasconi, Olivier J. F. Martin, "Modes interplay controls the second harmonic generation dynamics of plasmonic nanostructures," Proc. SPIE 11462, Plasmonics: Design, Materials, Fabrication, Characterization, and Applications XVIII, 114621W (20 August 2020); doi: $10.1117 / 12.2567966$ 


\title{
Modes interplay controls the second harmonic generation dynamics of plasmonic nanostructures
}

\author{
Andrei Kiselev*, Gabriel D. Bernasconi, and Olivier J. F. Martin ${ }^{\dagger}$ \\ Nanophotonics and Metrology Laboratory, Swiss Federal Institute of Technology Lausanne (EPFL), \\ 1015 Lausanne, Switzerland
}

\begin{abstract}
A large amount of experimental and theoretical works deals with the second harmonic generation from different plasmonic geometries. Since they often consider relatively long optical pulses, many of these studies are focused on the investigation of a quasi-monochromatic response of the system and can be understood through the excitation of one, possibly two, optical modes. On the other hand, when the excitation pulse duration is short (say, below several tens of fs), the excitation spectrum becomes broader and a very interesting dynamics emerges from the interplay between several optical modes.

In this work, the dynamics of modes at the second harmonic frequency for two silver spheres of different diameters and a nanorod is investigated numerically and shown to be quite different. For the pulsed illumination with length close to the modes lifetime, apart from different relative contributions of dipolar and quadrupolar multipoles in the far-field, we have been able to observe and explain non constant phase difference between multipoles, which is not accessible in continuous wave regime. Short pulse durations also allow us to observe only one mode, while another one has already decayed. For the case of the nanorod we also perform an eigenmode analysis, which allows to understand the modes interplay that explains the observed spectra.

In the paper, we also show a method allowing a significant reduction of required computational steps to find the response of a plasmonic nanostructure to a pulsed illumination with arbitrary frequency-domain method.
\end{abstract}

Keywords: SHG, SFG, eigenmodes, surface integral equation method, multipoles, ultrafast photonics

\section{INTRODUCTION}

The invention of the first Ruby laser has enabled coherent light emission with extremely high intensities. ${ }^{1,2}$ The first experimental observation of the second harmonic generation (SHG) from quartz crystal was performed in the following years, ${ }^{3}$ giving inspiration to a plethora of theoretical studies on the topic. ${ }^{4,5}$ Peculiar properties associated with plasmonics, such as the ability to focus light beyond the diffraction limit and create high intensity hotspots, ${ }^{6,7}$ have attracted attention to second harmonic studies on plasmonic nanoparticles. ${ }^{8}$

Second harmonic generation is a nonlinear process that requires high laser intensities, usually achieved by pulsed illumination. Pulse compression down to hundreds of femtoseconds enables to observe second harmonic scattering with an average pulse power of several tens of milliwatts. Such relatively long pulses are spectrally narrow and can be considered as quasi-monochromatic, leading to the observation of the second harmonic $(\mathrm{SH})$ signal at single frequency; see theoretical ${ }^{9-15}$ and experimental ${ }^{16-23}$ publications on the topic. In the following, we will refer to such experiments as SHG. Interestingly, shrinking the pulse duration down to the lifetime of the modes supported by the nanostructure quite significantly changes the response. In addition to the interference between the modes ${ }^{23}$ already accessible for long pulses, the modes now have more complicated interplay dynamics due to the simultaneous contributions of different frequencies to the signal. ${ }^{24}$ These types of experiments will be referred to as sum frequency generation (SFG). For example, the effect, in which one mode has already decayed, while another one still radiates can be observed in that case. ${ }^{24}$ Consequently, the SH radiation directivity can vary in time in such experiments. ${ }^{23}$ In addition, experiments with ultrashort laser pulses have been recently conducted with the goal to compress the laser pulse duration below the Fourier limit. $^{25}$

*andrei.kiselev@epfl.ch, †olivier.martin@epfl.ch

Plasmonics: Design, Materials, Fabrication, Characterization, and Applications XVIII,

edited by Din Ping Tsai, Takuo Tanaka, Proc. of SPIE Vol. 11462, 114621W

(C) 2020 SPIE - CCC code: 0277-786X/20/\$21 - doi: 10.1117/12.2567966 
In this work, we focus on the study of the SH response of nanoparticles excited with ultrashort Gaussian pulses and study the interplay of various modes. We extend the previously published analysis ${ }^{26}$ by studying the influence of the morphology on the position of the SH resonances for two silver spheres of 60 and $120 \mathrm{~nm}$ in diameters and compare it to a nanorod with the size of $40 \times 40 \times 120 \mathrm{~nm}^{3}$. An elaborated analysis of the beating effects for different spectral components of dipolar mode for 20 and $40 \mathrm{~nm}$ spheres is also performed.

\section{METHODS}

In this paper, we perform a numerical study of the second harmonic response from small metallic nanoparticles with different forms and geometries, illuminated by a Gaussian pulse. For our simulation, we use the surface integral equation method based on the T-PMCHWT formulation, to calculate the linear ${ }^{27}$ and second harmonic ${ }^{28}$ scattering responses. We use the same methods for scattering calculations and mode analysis as discussed in Kiselev et al. ${ }^{26}$ Before proceeding to the results, we would like to discuss the method that allows speeding up the computation of the time-dynamic signal at the second harmonic frequency for pulsed illumination. As was pointed out, ${ }^{26}$ to calculate the second order time-domain nonlinear response in metals, one needs to take into account the SFG process, in which photons at the fundamental frequencies $\omega_{1}$ and $\omega_{2}$ combine together to create the local-surface polarization sources at the sum frequency $\Omega=\omega_{1}+\omega_{2} \cdot{ }^{29}$ The local-surface polarization sources can be found as

$$
P_{n}^{(2)}(\mathbf{r}, \Omega)=\chi_{n, n n}^{(2)} \sum_{\omega} E_{n}(\mathbf{r}, \omega) E_{n}(\mathbf{r}, \Omega-\omega),
$$

where $E_{n}(\mathbf{r}, \omega)$ is the electric field's normal component just below the surface at the position $\mathbf{r}$ and $\chi_{n, n n}^{(2)}$ is a normal component of the surface tensor polarizability. Each point of the surface of the scatterer then can be considered as the source of the second harmonic signal and the self-consistent second harmonic response can be obtained. ${ }^{27}$ From the numerical calculation perspective, finding the second harmonic response can be represented as a solution of a matrix equation

$$
\mathbf{C}^{(\Omega)} \cdot \mathbf{x}^{(\Omega)}=\mathbf{y}^{(\Omega)}
$$

Here $\mathbf{C}^{(\Omega)}$ is the SFG matrix, ${ }^{27} \mathbf{x}^{(\Omega)}$ is the vector representing the distribution of self-consistent electric field on the surface of the scatterer and $\mathbf{y}^{(\Omega)}$ is the vector containing information about the surface polarization sources. Assuming that the pulse contains a set of $\mathrm{N}$ frequencies $\omega_{n}, n=1 . . N$, Eq. (2) needs to be solved for all frequency pairs $\left(\omega_{i}, \omega_{j}\right)$. The most resource-consuming procedures are related to filling the SFG matrix $\mathbf{C}^{(\Omega)}$, which requires $\left(N^{2}+N\right) / 2$ operations. ${ }^{24}$ However, the $\mathbf{C}^{(\Omega)}$ matrix depends only on the sum frequency $\Omega$ and does not depend on the constituting linear-signal frequencies $\omega_{i}+\omega_{j}=\Omega$. This enables simplification of the calculations by storing the filled SFG matrix for the groups of frequencies leading to the same sum-frequency $\Omega$, thus reducing the required number of operations to $2 N+1$.

In our simulations, we have chosen silver as a material of study. It has lower losses and, consequently, increased lifetime for the plasmonic modes. The permittivity function was found by interpolating the experimental data of Johnson and Christy $^{30}$ to calculate scattering at the linear and second harmonic stages. A Drude model was used to calculate the eigenmodes with $\omega_{p}=9.3 \mathrm{eV}, \gamma=0.03 \mathrm{eV}, \varepsilon_{\infty}=4.3$ for $\omega \rightarrow \infty$. Water background was considered, with refractive index $n=1.33$.

\section{RESULTS}

\subsection{Second harmonic generation resonance shift due to size variation of spherical nanoparticles}

In this section, we study the influence of the size of a spherical metallic sphere on the positions and relative magnitudes of the resonances at the second harmonic response. Four silver metallic spheres with the diameters of 20, 40, 60 and 120 $\mathrm{nm}$ are considered and illuminated with a monochromatic planewave. The total scattering cross section along with the cross sections attributed to the electric dipolar and quadrupolar vector spherical harmonic contributions at the $\mathrm{SH}$ frequencies are presented in Figure 1 for the four sphere diameters. In the case of SH generation, the dipolar and 
quadrupolar contributions are attributed to the transversal dipolar (TD) and longitudinal quadrupolar modes (LQ) excited at the second harmonic frequency. ${ }^{11,23,26}$ The position of the resonances as well as the relative ratio between quadrupolar and dipolar peak values are listed in Table 1. The increase of the dipolar response for higher energies (up to $3.6 \mathrm{eV}$ and above) can be attributed to the second harmonic generation produced from the surface of the nanoparticles by bulk oscillations since the real part of the dielectric permittivity of silver becomes positive for energies higher than $4.5 \mathrm{eV}$. As can be seen from Table 1, the position of each resonance experiences a redshift as the sphere diameter increases, in agreement with Singh et al. ${ }^{31}$ For the $120 \mathrm{~nm}$ sphere, the dipolar resonance becomes very broad and it is difficult to assess its position accurately. We note it as "Undef." in Table 1. The relative contribution of the quadrupolar response also increases as experimentally shown in Butet et al. ${ }^{21}$ Thus, by increasing the size of the object, we increase the quadrupolar contribution but, on the other hand, decrease the energy of the resonances. In the following section, we show that the position of the quadrupolar resonances can be maintained at the same level as for a $20 \mathrm{~nm}$ sphere with significant increase of the quadrupolar response, by changing the geometry of the scatterer.
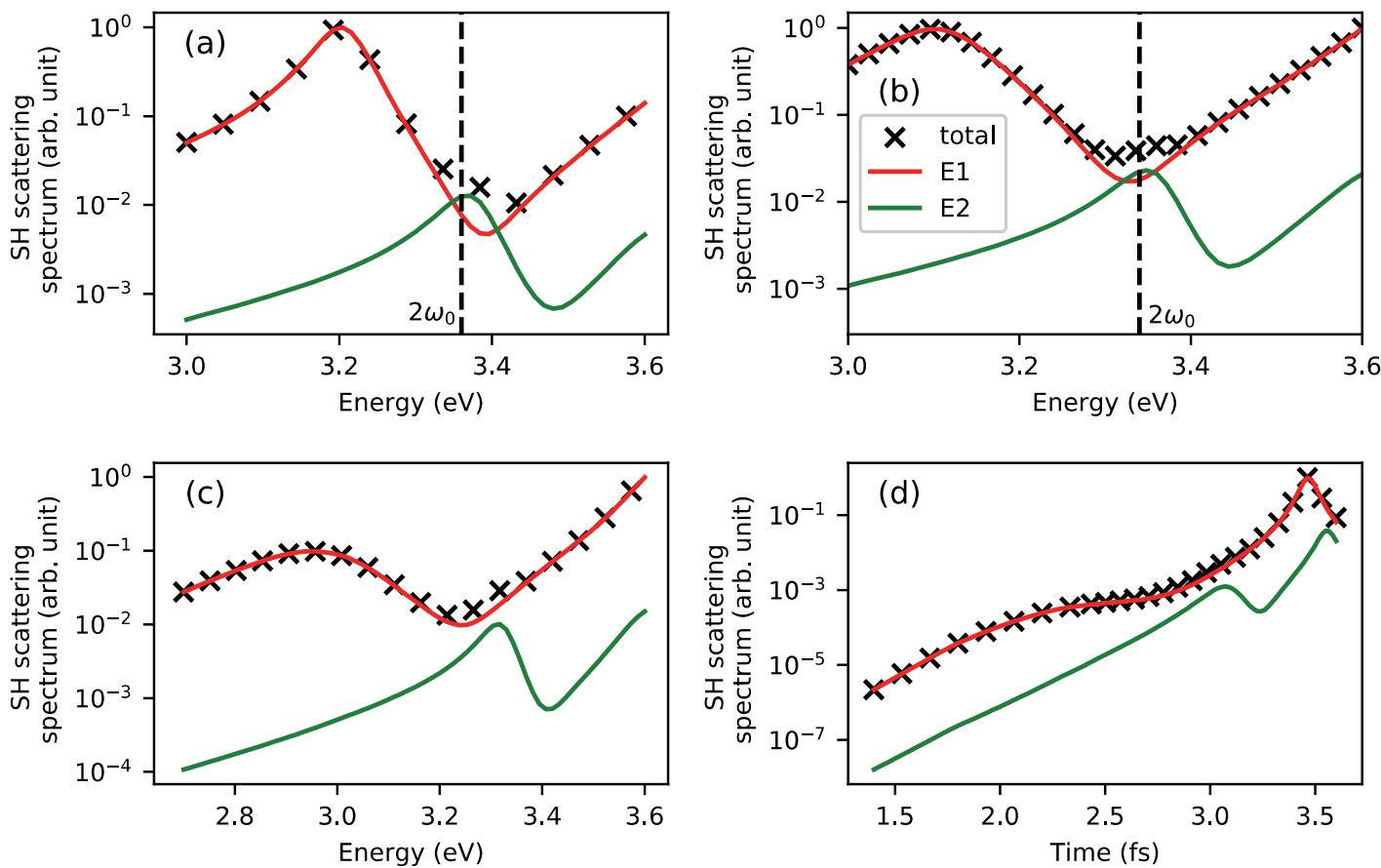

Figure 1. SHG for four silver spheres with different diameters. For all panels: black crosses stand for the total SH scattering cross section collected at all angles, red and green solid curves represent dipolar and quadrupolar contributions to the signal. (a) SH monochromatic spectrum for a $20 \mathrm{~nm}$ silver sphere. (b) SH monochromatic spectrum for a $40 \mathrm{~nm}$ silver sphere. (c) SH monochromatic spectrum for a $60 \mathrm{~nm}$ silver sphere. (d) SH monochromatic spectrum for a $120 \mathrm{~nm}$ silver sphere.

Table 1. Resonance positions for different sphere diameters.

\begin{tabular}{|c|c|c|c|}
\hline $\begin{array}{c}\text { Sphere } \\
\text { diameter, } \\
\mathrm{nm}\end{array}$ & $\begin{array}{c}\mathrm{TD}, \\
\hbar \omega(\mathrm{eV})\end{array}$ & $\begin{array}{c}\mathrm{LQ}, \\
\hbar \omega(\mathrm{eV})\end{array}$ & $\begin{array}{c}\mathrm{LQ} / \mathrm{TD} \\
\text { peak } \\
\text { amplitude } \\
\text { ratio }\end{array}$ \\
\hline 20 & 3.2 & 3.38 & 0.01 \\
\hline 40 & 3.1 & 3.34 & 0.023 \\
\hline 60 & 2.95 & 3.31 & 0.07 \\
\hline 120 & Undef. & 3.07 & Undef. \\
\hline
\end{tabular}




\subsection{Nanorod}

In this section, we consider a metallic nanorod with dimensions $40 \times 40 \times 120 \mathrm{~nm}^{3}$ and excited with a planewave propagating along its long axis. The second harmonic generation scattering spectrum along with the vector spherical harmonic contributions up to octupolar are presented in Figure 2. As can be seen, the position of the dipolar and quadrupolar resonances is now at $3.17 \mathrm{eV}$ and $3.3 \mathrm{eV}$, respectively, while the overall quadrupolar contribution is significantly enhanced, compared to the $20 \mathrm{~nm}$ sphere, Figure 1(a).

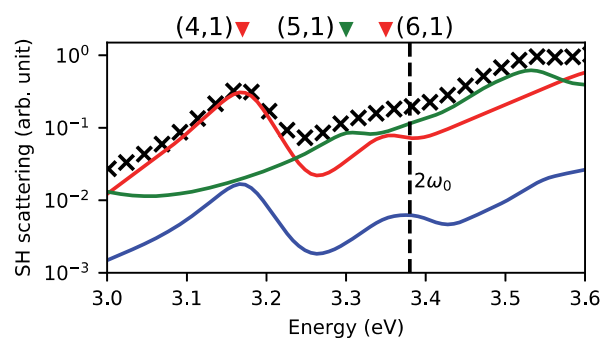

Figure 2. SHG signal for a silver nanorod. The black crosses, red, green and blue curves correspond to the total response and its dipolar, quadrupolar and octupolar components, respectively.

We performed an eigenmode analysis to reveal the origin of the peaks in the SH signal. The charge distributions for the modes responsible for the $\mathrm{SH}$ emission at different frequencies in the case of a nanorod are presented in Figure 3. Note that slight discrepancy (around $0.1 \mathrm{eV}$ ) between the eigenfrequencies and the positions of the peaks in Figure 2 is caused by an underestimation of the real part of the permittivity in the Drude model. Interestingly, the dipolar response is found for high order modes at 3.23 and $3.48 \mathrm{eV}$, Figure 3(a) and 3(c). A high quadrupolar response is observed for the mode at $3.41 \mathrm{eV}$, Figure 3(b). This effect is somewhat counterintuitive: one would expect a more complex far-field response from high-order modes as is the case for radio antenna theory. For example, the $(4,1)$ mode, Figure 3(b), would be expected to radiate mostly as an octupole. However, in plasmonics, for the modes at the second harmonic frequency this is not the case and high order modes can radiate with low-order vector spherical harmonics. A similar effect has been observed in linear scattering from plasmonic nanostructures, where it was shown that high order modes of nonspherical structures radiate with lower-order vector spherical harmonics. ${ }^{32}$

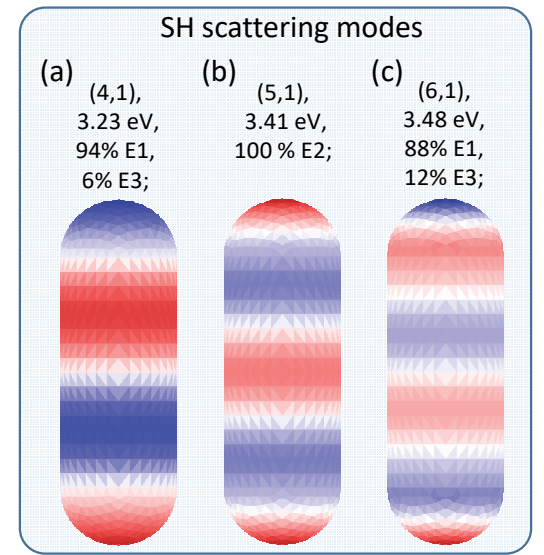

Figure 3. Charge distribution for the eigenmodes supported by the nanorod. The first two numbers in brackets characterize the mode by representing the number of charged poles along the long (first number) and the short (second number) axes of the rod. Red color stands for positive values of the charge and blue for negative. The value in $\mathrm{eV}$ represents the eigenfrequency of the mode. E1, E2 and E3 correspond to the relative contribution in the far-field of the electric dipolar, quadrupolar and octupolar spherical harmonics, respectively. 


\subsection{Silver sphere dynamics}

We now address the far-field scattering from nanostructures under ultrashort pulsed illumination. In this section, we study the dynamics of the second harmonic signal for nanoparticles immersed in water under a Gaussian pulsed illumination of the form

$$
\mathbf{E}(t, \mathbf{r})=\mathbf{A} \exp \left(-t^{2} /\left(2 \Delta t^{2}\right)\right) \cos \left(\omega_{0} t-\mathbf{k} \cdot \mathbf{r}\right) .
$$

Here $\Delta t$ is the pulse width, $\omega_{0}$ is the central frequency, $\mathbf{k}$ is the wave vector with $|\mathbf{k}|=\omega_{0} / c$ and $c$ is the speed of light in water. In our simulations, we use the width of the pulse $\Delta t=22 \mathrm{fs}$, equals to a spectral width of $0.071 \mathrm{eV}$. In Figure 4 , we present the dynamics of the signal for the scattering from a $20 \mathrm{~nm}$ sphere excited with a Gaussian pulse with the central frequency $\omega_{0}=3.36 / 2 \mathrm{eV}$ (dashed line at double frequency in Figure 4(a) and 1(a)). Despite the fact that the quadrupolar mode is leading at the excitation frequency of the monochromatic spectrum, Figure 1(a), the spectrum of the SFG signal, as well as the far-field signal in time-domain, have a strong dipolar response caused by the neighboring dipolar peak at $3.2 \mathrm{eV}$. Interestingly, the relative phase between dipolar and quadrupolar signals in this case changes with time due to different eigenfrequencies of the dipolar and quadrupolar modes. The dip in the dipolar signal in Figure 4(b) is attributed to the beating between dipolar components of the signal grouped close to $\sim 3.25 \mathrm{eV}$ and $\sim 3.46 \mathrm{eV}$. Indeed, the beating period $T_{\text {beat }}$ can be estimated as

$$
T_{\text {beat }} \sim \frac{2 \pi}{(3.25 \cdot \mathrm{eV}-3.46 \cdot \mathrm{eV}) n}=15 \mathrm{fs},
$$

while the distance between the peaks in Figure $4(\mathrm{~b})$ is $\sim 12 \mathrm{fs}$.
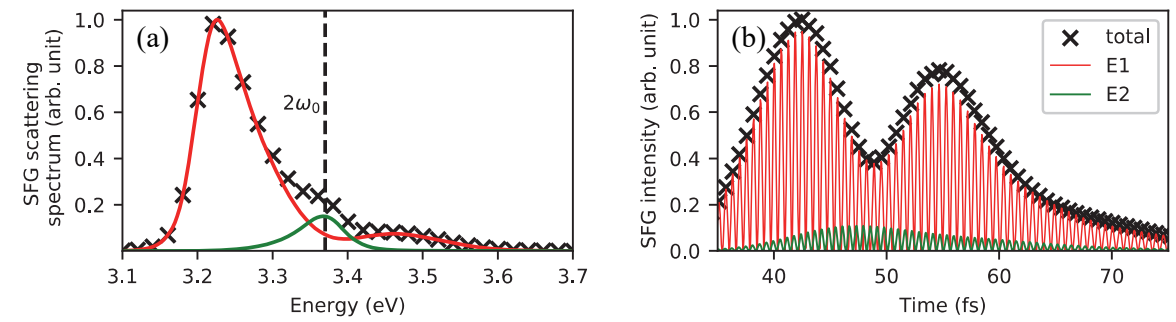

Figure 4. SFG for a $20 \mathrm{~nm}$ silver sphere. (a) Spectrum of the SFG signal for a Gaussian pulse excitation with $\omega_{0}=3.36 / 2 \mathrm{eV}$ and width $0.071 \mathrm{eV}$. (d) SFG far-field intensity dynamics.

We also study the SFG signal and dynamics of the SH signal from a $40 \mathrm{~nm}$ sphere excited with a Gaussian pulse with central frequency $\omega_{0}=3.34 / 2 \mathrm{eV}$ (dashed line at double frequency in Figure 5(a) and 1(b)). The results are presented in Figure 5. As seen from Figure 5(b), a pure quadrupolar signal is only obtained close to 50 fs with a duration of several femtoseconds. The dip in the SH time-domain signal is observed again, due to the beating between dipolar oscillations at frequencies close to $3.2 \mathrm{eV}$ and $3.45 \mathrm{eV}$. Interestingly, in the dynamics of the modes, the second peak at a later time close to $55 \mathrm{fs}$ has weaker amplitude compared to the one close to $42 \mathrm{fs}$. Based on the Mie solution, we made an analysis of the eigenfrequencies for 20 and $40 \mathrm{~nm}$ silver sphere. It was found that, apart from the variation of the resonance frequency, the imaginary part of the eigenfrequency also increases as the sphere becomes larger. Since the imaginary part of the eigenfrequency is responsible for mode's damping, an increment of the size of the nanostructure leads to higher losses, thus decreasing the height of the second peak in Figure 5(b). 

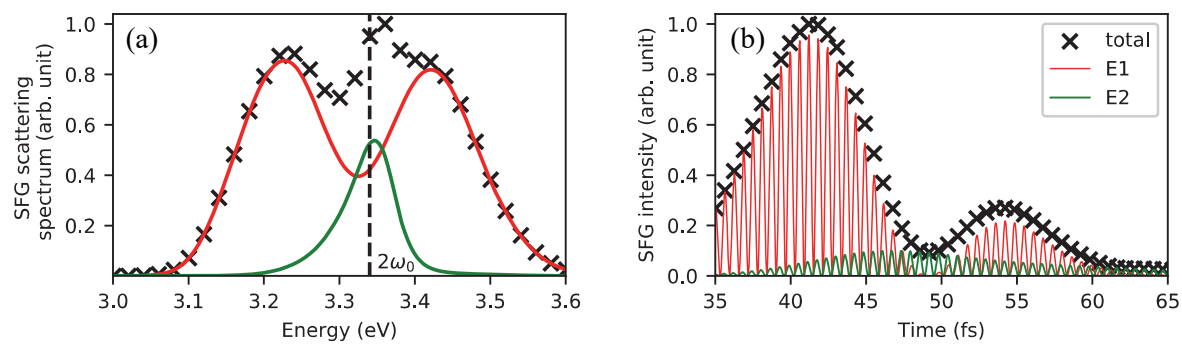

Figure 5. SFG for a $40 \mathrm{~nm}$ silver sphere. (a) Spectrum of the SFG signal for a Gaussian pulse excitation with $\omega_{0}=3.34 / 2 \mathrm{eV}$ and width $0.071 \mathrm{eV}$. (d) SFG far-field intensity dynamics.

To make a comparison between the dynamics of the sphere and that of a nanorod, in Figure 6 we present the dynamics of the second harmonic signal for the nanorod excited with a Gaussian pulse centered at 3.38/2 eV with the same pulse width as in Figure 5. As seen from Figure 6(a), the spectrum of the SHG response in this case is mostly quadrupolar. As a result, the SH signal in time-domain has also a quadrupolar response, Figure 6(b). Closer inspection of the dynamics of the different modes in Figure 6(b) reveals a barely varying phase shift between the dipolar, quadrupolar and octupolar modes, because the spectral components of these harmonics, Figure 6(a), have high amplitudes around the same energy. Due to the different lifetimes of the dipolar and quadrupolar modes ${ }^{24}$ the quadrupolar signal lasts longer in the pulse, while the dipolar mode decays earlier.
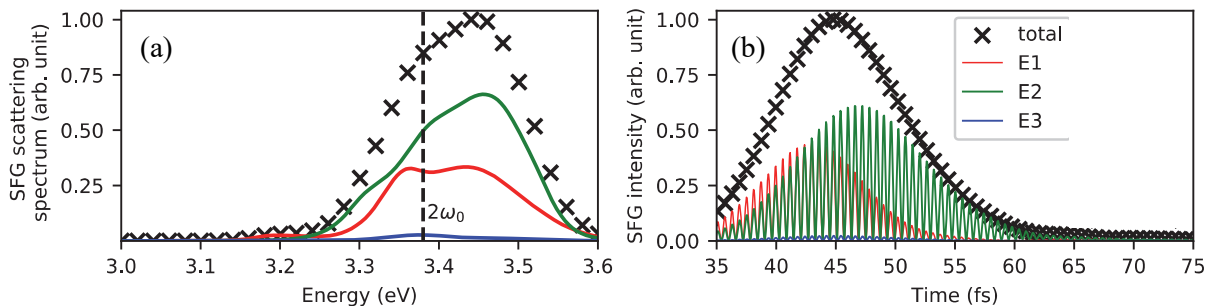

Figure 6. SFG for a $40 \times 40 \times 120 \mathrm{~nm}^{3}$ nanorod. (a) Spectrum of the SFG signal for a Gaussian pulse excitation with $\omega_{0}$ $=3.38 / 2 \mathrm{eV}$ and width $0.071 \mathrm{eV}$. (d) SFG far-field intensity dynamics.

\section{CONLUSIONS}

We have numerically studied quasi-monochromatic second harmonic scattering from 20, 40, 60 and 120 nm diameter spheres. It was shown that the position of dipolar and quadrupolar resonances experiences a redshift as the sphere diameter increases. The example of a nanorod has been presented to show that the position of the resonances can be tuned to appear close to those observed for a $20 \mathrm{~nm}$ sphere. In the same time, a nanorod radiates with significantly increased quadrupolar components, compared to the case of a sphere.

The dynamics of the second harmonic signal due to illumination with Gaussian pulses as short as 22 fs has been also presented and found very different for 20 and $40 \mathrm{~nm}$ spheres and for a nanorod. Based on the analysis of the linear second harmonic quasi-monochromatic features and plasmon lifetime in these structures, we have explained the origin of the beating effects and the effect of faster decay of one mode, while another one is still radiating. 


\section{ACKNOWLEDGMENTS}

Funding from the European Research Council (ERC-2015-AdG-695206) is gratefully acknowledged.

\section{REFERENCES}

1. T. H. Maiman, "Stimulated Optical Radiation in Ruby," Nature 187(4736), 493-494 (1960).

2. R. J. Collins, D. F. Nelson, A. L. Schawlow, W. Bond, C. G. B. Garrett, and W. Kaiser, "Coherence, Narrowing, Directionality, and Relaxation Oscillations in the Light Emission from Ruby," Phys. Rev. Lett. 5(7), 303-305 (1960).

3. P. A. Franken, A. E. Hill, C. W. Peters, and G. Weinreich, "Generation of Optical Harmonics," Phys. Rev. Lett. 7(4), 118-119 (1961).

4. R. W. Boyd, Nonlinear Optics (Academic, 2008).

5. Y.-R. Shen, The principles of nonlinear optics (Wiley-Interscience, 2002).

6. T. Siegfried, Y. Ekinci, O. J. F. Martin, and H. Sigg, "Gap Plasmons and Near-Field Enhancement in Closely Packed Sub-10 nm Gap Resonators," Nano Lett. 13(11), 5449-5453 (2013).

7. J. P. Kottmann, O. J. F. Martin, D. R. Smith, and S. Schultz, "Non-regularly shaped plasmon resonant nanoparticle as localized light source for near-field microscopy," J. Microsc. 202(1), 60-65 (2001).

8. J. Butet, P.-F. Brevet, and O. J. F. Martin, "Optical Second Harmonic Generation in Plasmonic Nanostructures: From Fundamental Principles to Advanced Applications," ACS Nano 9(11), 10545-10562 (2015).

9. Y. Pavlyukh and W. Hübner, "Nonlinear Mie scattering from spherical particles," Phys. Rev. B 70(24), 245434 (2004).

10. J. I. Dadap, J. Shan, K. B. Eisenthal, and T. F. Heinz, "Second-Harmonic Rayleigh Scattering from a Sphere of Centrosymmetric Material," Phys. Rev. Lett. 83(20), 4045-4048 (1999).

11. J. I. Dadap, J. Shan, and T. F. Heinz, "Theory of optical second-harmonic generation from a sphere of centrosymmetric material: small-particle limit," J. Opt. Soc. Am. B 21(7), 1328-1347 (2004).

12. A. Capretti, C. Forestiere, L. Dal Negro, and G. Miano, "Full-Wave Analytical Solution of Second-Harmonic Generation in Metal Nanospheres," Plasmonics 9(1), 151-166 (2014).

13. A. G. F. de Beer and S. Roke, "Nonlinear Mie theory for second-harmonic and sum-frequency scattering," Phys. Rev. B 79(15), 155420 (2009).

14. V. L. Brudny, B. S. Mendoza, and W. Luis Mochán, "Second-harmonic generation from spherical particles," Phys. Rev. B 62(16), 11152-11162 (2000).

15. G. Bachelier, I. Russier-Antoine, E. Benichou, C. Jonin, and P.-F. Brevet, "Multipolar second-harmonic generation in noble metal nanoparticles," J. Opt. Soc. Am. B 25(6), 955-960 (2008).

16. J. Nappa, G. Revillod, I. Russier-Antoine, E. Benichou, C. Jonin, and P. F. Brevet, "Electric dipole origin of the second harmonic generation of small metallic particles," Phys. Rev. B 71(16), 165407 (2005).

17. E. C. Hao, G. C. Schatz, R. C. Johnson, and J. T. Hupp, "Hyper-Rayleigh scattering from silver nanoparticles," J. Chem. Phys. 117(13), 5963-5966 (2002).

18. J. Shan, J. I. Dadap, I. Stiopkin, G. A. Reider, and T. F. Heinz, "Experimental study of optical second-harmonic scattering from spherical nanoparticles," Phys. Rev. A 73(2), 023819 (2006).

19. I. Russier-Antoine, E. Benichou, G. Bachelier, C. Jonin, and P. F. Brevet, "Multipolar Contributions of the Second Harmonic Generation from Silver and Gold Nanoparticles," J. Phys. Chem. C 111(26), 9044-9048 (2007).

20. J. Nappa, I. Russier-Antoine, E. Benichou, C. Jonin, and P. F. Brevet, "Second harmonic generation from small gold metallic particles: From the dipolar to the quadrupolar response," J. Chem. Phys. 125(18), 184712 (2006).

21. J. Butet, J. Duboisset, G. Bachelier, I. Russier-Antoine, E. Benichou, C. Jonin, and P.-F. Brevet, "Optical Second Harmonic Generation of Single Metallic Nanoparticles Embedded in a Homogeneous Medium," Nano Lett. 10(5), 1717 1721 (2010).

22. J. Butet, G. Bachelier, I. Russier-Antoine, C. Jonin, E. Benichou, and P. F. Brevet, "Interference between Selected Dipoles and Octupoles in the Optical Second-Harmonic Generation from Spherical Gold Nanoparticles," Phys. Rev. Lett. 105(7), 077401 (2010).

23. J. Butet, G. D. Bernasconi, M. Petit, A. Bouhelier, C. Yan, O. J. F. Martin, B. Cluzel, and O. Demichel, "Revealing a Mode Interplay That Controls Second-Harmonic Radiation in Gold Nanoantennas," ACS Photonics 4(11), 2923-2929 (2017). 
24. G. D. Bernasconi, J. Butet, and O. J. F. Martin, "Dynamics of Second-Harmonic Generation in a Plasmonic Silver Nanorod," ACS Photonics 5(8), 3246-3254 (2018).

25. N. Accanto, J. B. Nieder, L. Piatkowski, M. Castro-Lopez, F. Pastorelli, D. Brinks, and N. F. van Hulst, "Phase control of femtosecond pulses on the nanoscale using second harmonic nanoparticles," Light Sci. Appl. 3(1), e143-e143 (2014).

26. A. Kiselev, G. D. Bernasconi, and O. J. F. Martin, "Modes interplay and dynamics in the second harmonic generation of plasmonic nanostructures," Opt. Express 27(26), 38708-38720 (2019).

27. A. M. Kern and O. J. F. Martin, "Surface integral formulation for 3D simulations of plasmonic and high permittivity nanostructures," J. Opt. Soc. Am. A 26(4), 732-740 (2009).

28. J. Butet, B. Gallinet, K. Thyagarajan, and O. J. F. Martin, "Second-harmonic generation from periodic arrays of arbitrary shape plasmonic nanostructures: a surface integral approach," J. Opt. Soc. Am. B 30(11), 2970-2979 (2013).

29. Y. R. Shen, "Surface contribution versus bulk contribution in surface nonlinear optical spectroscopy," Appl. Phys. B 68(3), 295-300 (1999).

30. P. B. Johnson and R. W. Christy, "Optical Constants of the Noble Metals," Phys. Rev. B 6(12), 4370-4379 (1972).

31. A. Singh, A. Lehoux, H. Remita, J. Zyss, and I. Ledoux-Rak, "Second Harmonic Response of Gold Nanorods: A Strong Enhancement with the Aspect Ratio," J. Phys. Chem. Lett 4(22), 3958-3961 (2013).

32. J. Dorfmüller, R. Vogelgesang, W. Khunsin, C. Rockstuhl, C. Etrich, and K. Kern, "Plasmonic Nanowire Antennas: Experiment, Simulation, and Theory," Nano Lett. 10(9), 3596-3603 (2010). 